American Journal of Infectious Diseases 6 (4): 110-121, 2010

ISSN 1553-6203

(C) 2010 Science Publications

\title{
Antimicrobial Pattern and Clonal Dissemination of Extended-Spectrum Beta-Lactamase Producing Klebsiella Spp Isolates
}

\author{
${ }^{1}$ Sobhan Ghafourian, ${ }^{2}$ Nourkhoda Sadeghifard, ${ }^{1}$ Zamberi bin Sekawi, ${ }^{1}$ Vasantha Kumari Neela, \\ ${ }^{1}$ Mariana Nor Shamsudin, ${ }^{2}$ Reza Mohebi, ${ }^{3}$ Mohammad Rahbar and ${ }^{4}$ Mohammad Raftari \\ ${ }^{1}$ Department of Medical Microbiology and Parasitology, Faculty of Medicine and Science Health, \\ University Putra, Malaysia \\ ${ }^{2}$ Department of Medical Microbiology, Ilam university of Medical Sciences, Iran \\ ${ }^{3}$ Reference laboratory of Iran \\ ${ }^{4}$ Faculty of Food Science and Technology, University Putra Malaysia, Malaysia
}

\begin{abstract}
Problem statement: Gram-negative pathogens harboring ESBLs have caused numerous outbreaks of infections and are becoming an increasing therapeutic problem in many countries. The incidence of ESBL-producing strains among clinical isolates has been steadily increasing over the past years resulting in limitations of therapeutic option. The focus of this study was to examine the molecular epidemiology of ESBL-producing Klebsiella spp, investigate the susceptibility of Klebsiellae spp producing ESBLs towards non-beta-lactam antibiotics in the different seasons, identify the various clonal types of ESBL-producing K.pneumoniae and detect the dominant ESBL clonal types. Approach: Clinical isolates of Klebsiella spp were identified during the period March 2007April 2008. ESBLs production identified by phenotypic and genotypic methods. MLST was performed for dissemination of ESBLs producing K. pneumoniae. Results: The findings showed that 51.6\% of K.pneumoniae were produces ESBLs. 35.8, 21.2 and 38.7\% of K. pneumoniae producing ESBLs were resistant to amikacin, ciprofloxacin and cotrimoxazol, respectively. It was found that 40 and $27.3 \%$ of K.oxytoca producing ESBLs were resistant to cotrimoxazol and amikacin, respectively. The findings reflected that ESBLs existed in $73 \%$ of $\mathrm{K}$. oxytoca. The results showed that the frequency of blaSHV, blaTEM and blaCTX-M due to K.pneumoniae producing ESBLs were 87.5, 12.4 and 24.8\%, respectively. Of the eleven $K$. oxytoca producing ESBLs, $100 \%$ blaSHV were obtained. Based on the nucleotide variations of the five genetic loci, twenty-five different STs could be identified among thirty K.pneumoniae producing ESBLs isolates. Among the STs shared by multiple isolates, the most frequently encountered were 14, 16 and ST18. Conclusion: In conclusion, the percentage of K.oxytoca producing ESBLs was higher than K.pneumoniae producing ESBLs. Generally, K.penomoniae produces more ESBLs in winter and fall than in the other seasons.
\end{abstract}

Key words: Antimicrobial pattern, Klebsiella spp, Extended Spectrum Beta-Lactamases (ESBL), Klebsiella pneumoniae, Urinary Tract Infection (UTI), Respiratory Tract Infection (RTI), Intensive Care Units (ICUs), Single Locus Variant (SLV)

\section{INTRODUCTION}

In recent years, extended-spectrum beta-lactamaseproducing K.pneumoniae strains have become important pathogens in hospital-acquired infections, showing multi-resistance and causing more and more outbreaks in hospitals (Kamatchi et al., 2009). It is important to study the prevalence of $K$. pneumoniae in Milad Hospital in Tehran of Iran, as Iran is a fourseasoned country. K. pneumoniae is frequently isolated in the winter season and responsible for majority of the nosocomial infections.
The focus of this study was to examine the molecular epidemiology of ESBL-producing Klebsiella $s p p$, investigate the susceptibility of Klebsiellae spp producing ESBLs towards non-beta-lactam antibiotics in the different seasons, identify the various clonal types of ESBL-producing K.pneumoniae and detect the dominant ESBL clonal types.

\section{MATERIALS AND METHODS}

Bacterial isolates: Clinical isolates of Klebsiella spp were identified during the period March 2007-April

Corresponding Author: Zamberi bin Sekawi, Department of Medical Microbiology and Parasitology,

Faculty of Medicine and Science Health, University Putra, Malaysia 
Am. J. Infect. Dis., 6 (4): 110-121, 2010

Table 1: Primers for PCR

\begin{tabular}{llll}
\hline Primers & Sequence of primers & Size of amplicon & References \\
\hline Blatem & F: 5-GAGTATCAACATTTCCGTGTC-3 & $848 \mathrm{bp}$ & (Nasehi et al. (2010) \\
& R: 5-TAATCAGTGAGGCACCTTCTC-3 & & \\
Blashv & F: 5-AAGATCCACTATCGCCCAGCAG-3 & 231 bp & (Nasehi et al. (2010) \\
Blactx-m & R: 5-ATTCAGTTCCGTTCCCAGCGG-3 & & \\
& F:5-ACGCTGTTGTTAGGAAGTG-3 & 759bp & Mansouri and Ramazanzadeh (2009) \\
\hline
\end{tabular}

Table 2: Primers for MLST

\begin{tabular}{|c|c|c|c|}
\hline \multirow{2}{*}{$\begin{array}{l}\text { Primers } \\
\text { rpoB }\end{array}$} & Sequences & Refrences & Size \\
\hline & $\begin{array}{l}\text { RPOB1 CAG TTC CGC GTT GGC CTG } \\
\text { RPOB2 CGG AAC GGC CTG ACG TTG CAT }\end{array}$ & Mollet et al. (1997) & $687 \mathrm{bp}$ \\
\hline gyrA & $\begin{array}{l}\text { gyrA1 ATG AGC GAC CTT GCG AGA GAA AT } \\
\text { gyrA2 CTC GTC ACG CAG CGC GCT GAT GCC }\end{array}$ & Wertz et al. (2003) & $752 \mathrm{bp}$ \\
\hline gapA & $\begin{array}{l}\text { gapA1 AGA ACA TCA TCC CGT CCT CTA CC } \\
\text { gapA2 CCA GAA CTT TGT TGG AGT AAC C }\end{array}$ & Wertz et al. (2003) & $366 \mathrm{bp}$ \\
\hline gyrB & $\begin{array}{l}\text { gyrB1 GCC TCG AAA CCT TCA CCA } \\
\text { gyrB2 CGC GAC GTG CGG CCT CAC GG }\end{array}$ & Wertz et al. (2003) & $648 \mathrm{bp}$ \\
\hline $\begin{array}{l}\text { groEL } \\
\text { groEL2 }\end{array}$ & $\begin{array}{l}\text { groEL1 GAC GCT CGY GTR AAA ATG CTS C } \\
\text { GCA GTG CAA CTT TGA TAC CCA CG }\end{array}$ & Wertz et al. (2003) & $786 \mathrm{bp}$ \\
\hline
\end{tabular}

2008 in Milad hospital in tehran. The strains were isolated from urinary tract infections, ICUs, surgery wards, lesion and respiratory tract infection.

Detection of Klebsiella spp. producing ESBLs: The methods for the laboratory detection of ESBLs were based on recommendations from the Clinical and Laboratory Standard Institute (CLSI) and the Canadian External Quality Assessment Advisory Group for Antibiotic Resistance. However, there have been minor variations from these guidelines to suit the operations of laboratories in our settings. All the clinically significant isolates of Klebsiella spp. had to be tested against beta lactam drugs using a disc diffusion method. Any decrease in the zone sizes for the third - generation of cephalosporins had to be used as a criterion to test for ESBLs identification (CLSI, 2010).

ESBL screening methods: In-vitro sensitivity testing was performed using established CLSI procedure with ceftazidime (Ca) (30 ug), cefotaxime (Ce) (30 ug), ceftriaxone (Ci) (30 ug), azteronam (Ao) (30 ug) and cefpodoxime (Cep) (30 ug). The zone diameters were read using the revised NCCLS. Any zone diameter within the "grey zone" was considered a probable ESBL producing strain requiring phenotypic confirmatory testing (CLSI, 2010).

Phenotypic confirmatory method: Ceftazidime (30ug) versus ceftazidime/clavulanic (Cac) (30 10 $\mathrm{ug}^{-1}$ ), cefotaxime (30 ug) versus cefotaxime /clavulanic acid (Cec) (30 $\left.10 \mathrm{ug}^{-1}\right)$ and cefpodoxim versus cefpodoxim/clavulanic acid (Cep) (30 $\left.10 \mathrm{ug}^{-1}\right)$ were placed into a Muller-Hinton agar plate lawned with the test organism and incubated as described above. Regardless of the zone diameters, a $>5 \mathrm{~mm}$ increase in a zone diameter for an antimicrobial agent tested in combination with clavulanic acid versus its zone size when tested alone, indicated a probable ESBL production (CLSI, 2010). K.pneumoniae ATCC 700603 was used as a control for ESBL tests.

PCR amplification of blaTEM, blaSHV and blaCTX-M: The Polymerase Chain Reaction (PCR) was carried out by using primers in Table 1 . K.pneumoniae 7881 was used as a control for blagenes responsible for ESBLs production.

Effect of non beta-lactame antibiotics against $K$. pneumonia Producing ESBLs: Amikacin (Ak) (30 ug), cotrimoxazol (Co) (30 ug) ciprofloxacin (Cf) (30 ug), imipenem (I) (30 ug) were used among Klebsiella spp producing ESBLs towards non beta-lactam antibiotics (Sabrina et al., 2009 and Erum et al., 2010).

Multilocus Sequence Typing (MLST): Five housekeeping genes were selected to establish the MLST scheme for thirty $K$. pneumoniae in our study; rрoB (RNA polymerase -subunit), gyrA (DNA gyrase subunit A), gapA (glyceraldehydes 3-phosphate dehydrogenase A), groEL (GroEL protein) and gyrB (DNA gyrase subunit B) (Table 2). PCR amplifications were carried out under the following conditions: 35 cycles of denaturation at $94 \mathrm{c}$ for $30 \mathrm{sec}$, annealing at $50-55 \mathrm{c}$ for 30 second extension at $72 \mathrm{c}$ for $1 \mathrm{~min}$; the process was preceded by a 5 min denaturation at $94 \mathrm{c}$, followed by a $5 \mathrm{~min}$ final extension at $72 \mathrm{c}$. The PCR products were purified using a PCR purification kit (fermentase); then, the products were sequenced. The raw sequences were concatenated and edited by using the EditSeq and MegAlign programs. For each locus, distinct allele sequences were assigned as an arbitrary allele number. Each isolate was characterized by its 
allelic profile, represented as a series of five integers corresponding to the alleles at each of the loci, in the order of rpoB, gyrA, gapA, groEL and gyrB. The Sequence Type (ST) was designated for each unique allelic profile as a DNA start program. URL: (www.pubmlst.com).

Detection of bla TEM, SHV and CTX-M: To specify the subtype of the bla genes, the amplified PCR products were sequenced on both the strands as done in the MLST analysis. The amino acid sequences were deduced from the nucleotide sequences using the MegAlign program. They were compared with the database of the website (http://www.lahey.Org/ Studies/).

\section{RESULTS}

Of two hundred and eighty Klebsiella spp. Two hundred and sixty-five $K$. pneumoniae and fifteen K.oxytoca were obtained.

K.pneumoniae: In this study, generally, 65.8, 46.4, $60.4,52$ and $51.7 \%$ of the isolates were resistant to ceftazidime, cefotaxime, cefteriaxon, cefpodoxime and aztreonam, respectively (Table 3 ). The findings showed that $51.6 \%$ of K.pneumoniae produces ESBLs. Of the one hundred and thirty-seven K. pneumoniae producing ESBLs, 35.8, 21.2 and $38.7 \%$ were resistant to amikacin, ciprofloxacin and cotrimoxazol, respectively (Table 4). Of the K.pneumoniae suspected of producing ESBLs, 86.9\%, were confirmed by Ceftazidime/clavulanic acid, $31.4 \%$ by
Cefotaxime/clavulanic acid and $100 \%$ by Cefpodoxime/ clavulanic acid.

Screening stage: Of the two hundred and sixty-five $K$. pneumoniae collected in Milad hospital, 50.56\% ( $\mathrm{n}=$ 134), $12.07 \%(n=32), 9.82 \%(n=26), 8.68 \%(n=23)$ and $18.86 \%(\mathrm{n}=50)$ were from UTI, ICUs, surgery wards, lesion and respiratory tract infections, respectively.

As you can see in the Table 5, of the one hundred and thirty-four K.pneumoniae isolated from patients with UTI, it was found that there was more resistance to cefteriaxone than to the other beta-lactam antibiotics. The results also showed that sixty-eight isolates were suspected of being able to produce ESBLs. In the ICUs, thirty two K. pneumoniae showed the highest resistance to cefteriaxone $(59.4 \%)$ and cefatzidime $(56.25 \%)$. Generally, thirteen K. pneumoniae were suspected of being able to produce ESBLs (Table 6). Twenty six $K$. pneumoniae were obtained from patients admitted in surgery wards. Generally, resistance to ceftazidime $(80.4 \%)$ was not comparable to the other antibiotics. The findings revealed that eleven $K$. pneumoniae were prone to ESBLs production (Table 7). Twenty-two $K$. pneumoniae were obtained from patients with lesion infections, of which $34.8 \%$ were suspected of being able to produce ESBLs (Table 8). RTI had specified fifty $K$. pneumoniae (Table 9).

Confirming stage: All K. pnemoniae suspected to produce ESBLs had confirmed as showed in the Tables 8-12 in the confirming stage.

Table 3: Screening stage of all K.pneumoniae in all parts of Milad hospital

\begin{tabular}{lllllll}
\hline Milad hospital & K.pneumoniae & Ceftazidime & Cefotaxime & Cefteriaxone & Cefpodoxime & Aztreonam \\
\hline Total & 265 & 170 & 125 & 163 & 138 & 137 \\
& & $(65.8 \%)$ & $(46.4 \%)$ & $(60.4 \%)$ & $(52 \%)$ & $(51 \%)$ \\
\hline
\end{tabular}

Table 4: Effect of non-beta-lactam antibiotics against K.pneumoniae producing ESBLs in all parts of Milad hospital

\begin{tabular}{lllll}
\hline & K.pneumoniae producing ESBLs & Amikacine & Ciprofloxacine & Cotrimoxazol \\
\hline Total & 137 & 49 & 29 & 53 \\
& & $(35.8 \%)$ & $(21.2 \%)$ & $(38.7 \%)$ \\
\hline
\end{tabular}

Table 5: Screening stage of K.pneumoniae isolated from patients with UTI

\begin{tabular}{lllllll}
\hline UTI & K.pneumoniae & Ceftazidime & Cefotaxime & Cefteryaxone & Cefpodoxime & Aztreonam \\
\hline Spring & 31 & 11 & 19 & 18 & 16 & 16 \\
& $(23.14 \%)$ & $(35.48 \%)$ & $(61.29 \%)$ & $(58.06 \%)$ & $(51.61 \%)$ & $(51.61 \%)$ \\
Summer & 21 & 9 & 2 & 8 & 5 & 5 \\
& $(15.67 \%)$ & $(42.85 \%)$ & $(9.52 \%)$ & $(38.09 \%)$ & $(23.8 \%)$ & $(23.8 \%)$ \\
Fall & 39 & 21 & 15 & 19 & 18 & 18 \\
& $(29.1 \%)$ & $(53.85 \%)$ & $(38.46 \%)$ & $(48.71 \%)$ & $(46.15 \%)$ & $(46.15 \%)$ \\
Winter & 43 & 32 & 18 & 32 & 29 & 29 \\
& $(32.08 \%)$ & $(74.41 \%)$ & $(41.86 \%)$ & $(74.41 \%)$ & $(67.44 \%)$ & $(67.44 \%)$ \\
Total & 134 & 73 & 54 & $77 \%)$ & 68 & $(50.74 \%)$ \\
& $(100 \%)$ & $(54.4 \%)$ & $(40.3 \%)$ & $(57.5 \%)$ & $(50.74 \%)$ & \\
\hline
\end{tabular}


Am. J. Infect. Dis., 6 (4): 110-121, 2010

Table 6: Screening stage of K. pneumoniae of admitted patients in ICUs ward

\begin{tabular}{|c|c|c|c|c|c|c|}
\hline ICUs & K.pneumoniae & Ceftazidime & Cefotaxime & Cefteryaxone & Cefpodoxime & Aztreonam \\
\hline \multicolumn{7}{|l|}{ Ward } \\
\hline Spring & $\begin{array}{l}6 \\
(18.75 \%)\end{array}$ & $\begin{array}{l}3 \\
(50 \%)\end{array}$ & $\begin{array}{l}5 \\
(83.3 \%)\end{array}$ & $\begin{array}{l}4 \\
(66.7 \%)\end{array}$ & $\begin{array}{l}2 \\
(33.3 \%)\end{array}$ & $\begin{array}{l}2 \\
(33.3 \%)\end{array}$ \\
\hline Summer & $\begin{array}{l}7 \\
(21.88 \%)\end{array}$ & $\begin{array}{l}2 \\
(28.57 \%)\end{array}$ & $\begin{array}{l}1 \\
(14.28 \%)\end{array}$ & $\begin{array}{l}3 \\
(42.85 \%)\end{array}$ & $\begin{array}{l}3 \\
(42.85 \%)\end{array}$ & $\begin{array}{l}2 \\
(28.57 \%)\end{array}$ \\
\hline Fall & $\begin{array}{l}10 \\
(31.25 \%)\end{array}$ & $\begin{array}{l}7 \\
(70 \%)\end{array}$ & $\begin{array}{l}3 \\
(30 \%)\end{array}$ & $\begin{array}{l}6 \\
(60 \%)\end{array}$ & $\begin{array}{l}4 \\
(40 \%)\end{array}$ & $\begin{array}{l}4 \\
(40 \%)\end{array}$ \\
\hline Winter & $\begin{array}{l}9 \\
(28.1 \%)\end{array}$ & $\begin{array}{l}6 \\
(66.7 \%)\end{array}$ & $\begin{array}{l}4 \\
(44.5 \%)\end{array}$ & $\begin{array}{l}6 \\
(66.7 \%)\end{array}$ & $\begin{array}{l}5 \\
(55.5 \%)\end{array}$ & $\begin{array}{l}5 \\
(55.5 \%)\end{array}$ \\
\hline Total & $\begin{array}{l}32 \\
(100 \%) \\
\end{array}$ & $\begin{array}{l}18 \\
(56.25 \%)\end{array}$ & $\begin{array}{l}13 \\
(40.62 \%) \\
\end{array}$ & $\begin{array}{l}19 \\
(59.4 \%) \\
\end{array}$ & $\begin{array}{l}14 \\
(43.75 \%)\end{array}$ & $\begin{array}{l}13 \\
(40.62 \%) \\
\end{array}$ \\
\hline
\end{tabular}

Table 7: Screening stage of K. pneumoniae collected of admitted patients in surgery ward

\begin{tabular}{lllllll}
\hline Surgery ward & K.pneumoniae & Ceftazidime & Cefotaxime & Cefteriaxone & Cefpodoxime & Aztreonam \\
\hline Spring & 5 & 2 & 0 & 1 & 1 & 1 \\
& $(19.23 \%)$ & $(40 \%)$ & & $(20 \%)$ & $(20 \%)$ & $(20 \%)$ \\
Summer & 7 & 7 & 5 & 6 & 4 & 4 \\
\multirow{2}{*}{ Fall } & $(29.92 \%)$ & $(100 \%)$ & $(71.4 \%)$ & $(85.74 \%)$ & $(57.14 \%)$ & $(57.14 \%)$ \\
& 7 & 5 & 4 & 4 & 2 & 2 \\
Winter & $(29.92 \%)$ & $(71.4 \%)$ & $(57.14 \%)$ & $(57.14 \%)$ & $(28.57 \%)$ & $(28.57 \%)$ \\
& 7 & $(100 \%)$ & 4 & 5 & 4 & 4 \\
\multirow{2}{*}{ Total } & $(29.92 \%)$ & 21 & $(57.14 \%)$ & $(71.4 \%)$ & $(57.14 \%)$ & $(57.14 \%)$ \\
& 26 & $(80.4 \%)$ & $(50 \%)$ & $(61.5 \%)$ & $(42.3 \%)$ & $(42.3 \%)$ \\
\hline
\end{tabular}

Table 8: Screening stage of K.pneumoniae isolated of patients with lesion infection

\begin{tabular}{|c|c|c|c|c|c|c|}
\hline Lesion infection & K.Pneumoniae & Ceftazidime & Cefotaxime & Cefteryaxone & Cefpodoxime & Aztreonam \\
\hline Spring & $\begin{array}{l}5 \\
(21.7 \%)\end{array}$ & $\begin{array}{l}3 \\
(60 \%)\end{array}$ & $\begin{array}{l}1 \\
(20 \%)\end{array}$ & $\begin{array}{l}3 \\
(60 \%)\end{array}$ & $\begin{array}{l}2 \\
(40 \%)\end{array}$ & $\begin{array}{l}2 \\
(40 \%)\end{array}$ \\
\hline Summer & $\begin{array}{l}4 \\
(17.8 \%)\end{array}$ & $\begin{array}{l}3 \\
(75 \%)\end{array}$ & $(25 \%)$ & 2 & $(25 \%)$ & $\begin{array}{l}1 \\
(25 \%)\end{array}$ \\
\hline Fall & $\begin{array}{l}6 \\
(26.1 \%)\end{array}$ & $\begin{array}{l}4 \\
(66.7 \%)\end{array}$ & $\begin{array}{l}2 \\
(33.4 \%)\end{array}$ & $\begin{array}{l}4 \\
(66.7 \%)\end{array}$ & $\begin{array}{l}2 \\
(33.4 \%)\end{array}$ & $\begin{array}{l}2 \\
(33.4 \%)\end{array}$ \\
\hline Winter & $\begin{array}{l}8 \\
(34.78 \%)\end{array}$ & $\begin{array}{l}7 \\
(87.5 \%)\end{array}$ & 3 & $\begin{array}{l}4 \\
(50 \%)\end{array}$ & 3 & $\begin{array}{l}3 \\
(37.5 \%)\end{array}$ \\
\hline Total & $\begin{array}{l}23 \\
(100 \%)\end{array}$ & $\begin{array}{l}17 \\
(73.9 \%)\end{array}$ & $\begin{array}{l}7 \\
(30.4 \%)\end{array}$ & $\begin{array}{l}13 \\
(56.52 \%)\end{array}$ & $\begin{array}{l}8 \\
(34.8 \%)\end{array}$ & $\begin{array}{l}8 \\
(34.8 \%)\end{array}$ \\
\hline
\end{tabular}

Effects of non-beta-lactam antibiotics against $K$. pneumoniae producing ESBLs: In all the seasons, we found that $K$. pneumoniae producing ESBLs isolated from patients with UTI were resistant towards non-beta lactam antibiotics, but there were no resistance for imipenem (Table 10). In the ICUs, no resistance was observed in summer. In this part, also cotrimoxazol was found to be the most resistant antibiotics (Table 11). The findings showed that the K. pneumoniae producing ESBLs isolated from patients admitted in the surgery ward were not resistant to non-beta-lactam antibiotics in spring. The results also showed that there were more resistance to cotrimoxazol than to ciprofloxacin. Imipenem, as in the other parts was found to be an effective antibiotic (Table 12). Lesion infections did not show any resistance to imipenem and ciprofloxacin. It was also observed that there was no resistance in summer (Table 13). The results showed that ESBLs, produced by K.pneumoniae isolated from patients with RTI, were resistant to non-beta-lactam antibiotics except imipenem, in all the seasons (Table 14).

PCR results: Of the one hundred and twenty $K$. pneumoniae with blaSHV, 47.5\% $(\mathrm{n}=57), 9.1 \%(\mathrm{n}=$ $11), 9.1 \%(\mathrm{n}=11), 6.7 \%(\mathrm{n}=8)$ and $27.5 \%(\mathrm{n}=33)$ were from patients with urinary tract infections, patients admitted in ICUs, surgery ward, patients with lesion infections and patients with respiratory tract infections, respectively. Of the seventeen $K$. pneumoniae with blaTEM, 23.5\% $(\mathrm{n}=4), 11.7 \%(\mathrm{n}=$ 2), $11.7 \%(n=2), 5.9 \%(n=1)$ and $47 \%(n=8)$ were from patients with UTI, patients admitted in ICUs, surgery ward, patients with lesion infections and patients with RTIs, respectively. 
Am. J. Infect. Dis., 6 (4): 110-121, 2010

Table 9: Screening Stage of K. pneumoniae isolated from patients with RTI

\begin{tabular}{lllllll}
\hline RTI & K.Pneumonia & Ceftazidime & Cefotaxime & Cefteryaxone & Cefpodoxime & Aztreonam \\
\hline Spring & 9 & 8 & 8 & 7 & 7 & 7 \\
& $(18 \%)$ & $(88.9 \%)$ & $(88.9 \%)$ & $(77.8 \%)$ & $(77.8 \%)$ & $(77.8 \%)$ \\
Summer & 5 & 2 & 3 & 3 & 2 & 2 \\
& $(10 \%)$ & $(40 \%)$ & $(60 \%)$ & $(60 \%)$ & $(40 \%)$ & $(40 \%)$ \\
Fall & 12 & 11 & 9 & 9 & 8 & 8 \\
& $(24 \%)$ & $(91.7 \%)$ & $(75 \%)$ & $(75 \%)$ & $(66.7 \%)$ & $(66.7 \%)$ \\
\multirow{2}{*}{ Winter } & 24 & 20 & 18 & 19 & 20 & 20 \\
& $(48 \%)$ & $(83.3 \%)$ & $(75 \%)$ & $(79.1 \%)$ & $(83.3 \%)$ & $(83.3 \%)$ \\
\multirow{2}{*}{ Total } & 50 & $(11 \%)$ & 38 & 38 & 37 & 37 \\
& $(100 \%)$ & $(82 \%)$ & $(76 \%)$ & $(76 \%)$ & $(74 \%)$ & $(74 \%)$ \\
\hline
\end{tabular}

Table 10: Confirming stage and effect of non-beta-lactam antibiotics toward K.pnemoniae producing ESBLs from patients with UTI

\begin{tabular}{|c|c|c|c|c|c|c|c|c|}
\hline UTI & $\begin{array}{l}\text { K.pneumoniae } \\
\text { Suspected }\end{array}$ & $\begin{array}{l}\text { Ceftazidime/ } \\
\text { clavulanic acid }\end{array}$ & $\begin{array}{l}\text { Cefotaxime/ } \\
\text { clavulanicacid }\end{array}$ & $\begin{array}{l}\text { Cefpodoxime } \\
\text { clavulanicacid }\end{array}$ & Amikacine & $\begin{array}{l}\text { Ciproflox } \\
\text { acine }\end{array}$ & Cotrimoxazol & Imipenem \\
\hline & toproduce ESBLs & & & & & & & \\
\hline Spring & $\begin{array}{l}16 \\
(23.5 \%)\end{array}$ & $\begin{array}{l}16 \\
(100 \%)\end{array}$ & $\begin{array}{l}4 \\
(25 \%)\end{array}$ & $\begin{array}{l}16 \\
(100 \%)\end{array}$ & $\begin{array}{l}5 \\
(31.25 \%)\end{array}$ & $\begin{array}{l}4 \\
(25 \%)\end{array}$ & $\begin{array}{l}6 \\
(37.5 \%)\end{array}$ & 0 \\
\hline Summer & $\begin{array}{l}5 \\
(7.35 \%)\end{array}$ & $\begin{array}{l}5 \\
(100 \%)\end{array}$ & $\begin{array}{l}2 \\
(40 \%)\end{array}$ & $\begin{array}{l}5 \\
(100 \%)\end{array}$ & $\begin{array}{l}2 \\
(40 \%)\end{array}$ & $\begin{array}{l}1 \\
(20 \%)\end{array}$ & $\begin{array}{l}2 \\
(40 \%)\end{array}$ & 0 \\
\hline Fall & $\begin{array}{l}18 \\
(26.4 \%)\end{array}$ & $\begin{array}{l}16 \\
(88.9 \%)\end{array}$ & $\begin{array}{l}3 \\
(16.7 \%)\end{array}$ & $\begin{array}{l}18 \\
(100 \%)\end{array}$ & $\begin{array}{l}6 \\
(33.3 \%)\end{array}$ & $\begin{array}{l}5 \\
(27.8 \%)\end{array}$ & $\begin{array}{l}9 \\
(50 \%)\end{array}$ & 0 \\
\hline Winter & $\begin{array}{l}29 \\
(42.65 \%)\end{array}$ & $\begin{array}{l}21 \\
(72 \%)\end{array}$ & $\begin{array}{l}9 \\
(31 \%)\end{array}$ & $\begin{array}{l}29 \\
(100 \%)\end{array}$ & $\begin{array}{l}12 \\
(41.4 \%)\end{array}$ & $\begin{array}{l}6 \\
(20.7 \%)\end{array}$ & $\begin{array}{l}9 \\
(31 \%)\end{array}$ & 0 \\
\hline Total & $\begin{array}{l}68 \\
(100 \%)\end{array}$ & $\begin{array}{l}58 \\
(85.5 \%)\end{array}$ & $\begin{array}{l}18 \\
(26.47 \%)\end{array}$ & $\begin{array}{l}68 \\
(100 \%)\end{array}$ & $\begin{array}{l}25 \\
(36.7 \%)\end{array}$ & $\begin{array}{l}16 \\
(23.5 \%)\end{array}$ & $\begin{array}{l}26 \\
(38.2 \%)\end{array}$ & 0 \\
\hline
\end{tabular}

Table 11: Confirming stage and effect of non-beta-lactam antibiotics toward K.pnemoniae producing ESBLs isolated from patients in ICUs ward

\begin{tabular}{|c|c|c|c|c|c|c|c|c|}
\hline ICUS Ward & $\begin{array}{l}\text { K.pneumoniae } \\
\text { suspected to }\end{array}$ & $\begin{array}{l}\text { Ceftazidime/ } \\
\text { clavulanic acid }\end{array}$ & $\begin{array}{l}\text { Cefotaxime/ } \\
\text { clavulanicacid }\end{array}$ & $\begin{array}{l}\text { Cefpodoxime/ } \\
\text { clavulanicacid }\end{array}$ & Amikacine & Ciprofloxacine & Cotrimoxazol & Imipenem \\
\hline & produce ESBLs & & & & & & & \\
\hline Spring & $\begin{array}{l}2 \\
(15.4 \%)\end{array}$ & $\begin{array}{l}2 \\
(100 \%)\end{array}$ & $\begin{array}{l}1 \\
(50 \%)\end{array}$ & $\begin{array}{l}2 \\
(100 \%)\end{array}$ & $\begin{array}{l}1 \\
(50 \%)\end{array}$ & $\begin{array}{l}1 \\
(50 \%)\end{array}$ & $\begin{array}{l}1 \\
(50 \%)\end{array}$ & 0 \\
\hline Summer & $\begin{array}{l}2 \\
(15.4 \%)\end{array}$ & $\begin{array}{l}1 \\
(50 \%)\end{array}$ & $\begin{array}{l}1 \\
(50 \%)\end{array}$ & $\begin{array}{l}2 \\
(100 \%)\end{array}$ & 0 & 0 & 0 & 0 \\
\hline Fall & $\begin{array}{l}4 \\
(30.8 \%)\end{array}$ & $\begin{array}{l}3 \\
(75 \%)\end{array}$ & $\begin{array}{l}1 \\
(25 \%)\end{array}$ & $\begin{array}{l}4 \\
(100 \%)\end{array}$ & $\begin{array}{l}1 \\
(25 \%)\end{array}$ & $\begin{array}{l}1 \\
(25 \%)\end{array}$ & $\begin{array}{l}1 \\
(25 \%)\end{array}$ & 0 \\
\hline Winter & $\begin{array}{l}5 \\
(38.4 \%)\end{array}$ & $\begin{array}{l}5 \\
(100 \%)\end{array}$ & $\begin{array}{l}2 \\
(40 \%)\end{array}$ & $\begin{array}{l}5 \\
(100 \%)\end{array}$ & $\begin{array}{l}2 \\
(40 \%)\end{array}$ & $\begin{array}{l}1 \\
(20 \%)\end{array}$ & $\begin{array}{l}3 \\
(60 \%)\end{array}$ & 0 \\
\hline Total & $\begin{array}{l}13 \\
(100 \%)\end{array}$ & $\begin{array}{l}11 \\
(84.6 \%)\end{array}$ & $\begin{array}{l}5 \\
(38.46 \%)\end{array}$ & $\begin{array}{l}13 \\
(100 \%)\end{array}$ & $\begin{array}{l}4 \\
(30.7 \%)\end{array}$ & $\begin{array}{l}3 \\
(23 \%)\end{array}$ & $\begin{array}{l}5 \\
(38.46 \%)\end{array}$ & 0 \\
\hline
\end{tabular}

Table 12: Confirming stage and effect of non-beta-lactam antibiotics toward K.pnemoniae producing ESBLs isolated from patients in surgery

\begin{tabular}{|c|c|c|c|c|c|c|c|c|}
\hline Surgery ward & $\begin{array}{l}\text { K.pneumoniae } \\
\text { /clavulanic acid }\end{array}$ & $\begin{array}{l}\text { Ceftazidime } \\
\text { /clavulanicacid }\end{array}$ & $\begin{array}{l}\text { Cefotaxime } \\
\text { /clavulanicacid }\end{array}$ & Cefpodoxime & Amikacine & Ciprofloxacine & Cotrimoxazol & Imipenem \\
\hline Spring & $\begin{array}{l}1 \\
(9.1 \%)\end{array}$ & $\begin{array}{l}1 \\
(100 \%)\end{array}$ & $\begin{array}{l}0 \\
(100 \%)\end{array}$ & 1 & 0 & 0 & 0 & 0 \\
\hline Summer & $\begin{array}{l}4 \\
(36.4 \%)\end{array}$ & $\begin{array}{l}4 \\
(100 \%)\end{array}$ & $\begin{array}{l}1 \\
(25 \%)\end{array}$ & $\begin{array}{l}4 \\
(100 \%)\end{array}$ & $\begin{array}{l}1 \\
(25 \%)\end{array}$ & $\begin{array}{l}0 \\
(50 \%)\end{array}$ & 2 & 0 \\
\hline Fall & $\begin{array}{l}2 \\
(18.2 \%)\end{array}$ & $\begin{array}{l}2 \\
(100 \%)\end{array}$ & $\begin{array}{l}1 \\
(50 \%)\end{array}$ & $\begin{array}{l}2 \\
(100 \%)\end{array}$ & $\begin{array}{l}1 \\
(50 \%)\end{array}$ & $\begin{array}{l}1 \\
(50 \%)\end{array}$ & $\begin{array}{l}1 \\
(50 \%)\end{array}$ & 0 \\
\hline Winter & $\begin{array}{l}4 \\
(36.4 \%)\end{array}$ & $\begin{array}{l}4 \\
(100 \%)\end{array}$ & $\begin{array}{l}2 \\
(50 \%)\end{array}$ & $\begin{array}{l}4 \\
(100 \%)\end{array}$ & $\begin{array}{l}2 \\
(50 \%)\end{array}$ & $\begin{array}{l}1 \\
(25 \%)\end{array}$ & $\begin{array}{l}2 \\
(50 \%)\end{array}$ & 0 \\
\hline Total & $\begin{array}{l}11 \\
(100 \%)\end{array}$ & $\begin{array}{l}11 \\
(100 \%)\end{array}$ & $\begin{array}{l}4 \\
(36.6 \%)\end{array}$ & $\begin{array}{l}11 \\
(100 \%)\end{array}$ & $\begin{array}{l}4 \\
(36.6 \%)\end{array}$ & $\begin{array}{l}2 \\
(18.1 \%)\end{array}$ & $\begin{array}{l}5 \\
(45.5 \%)\end{array}$ & 0 \\
\hline
\end{tabular}


Am. J. Infect. Dis., 6 (4): 110-121, 2010

Table 13: Confirming stage and effect of non-beta-lactam antibiotics toward K.pnemoniae producing ESBLs isolated from patients with lesion infection

\begin{tabular}{|c|c|c|c|c|c|c|c|c|}
\hline Lesion infection & $\begin{array}{l}\text { K.pneumoniae } \\
\text { suspected to } \\
\text { produceESBLs }\end{array}$ & $\begin{array}{l}\text { Ceftazidime } \\
\text { /clavulanic acid }\end{array}$ & $\begin{array}{l}\text { Cefotaxime } \\
\text { /clavulanic acid }\end{array}$ & $\begin{array}{l}\text { Cefpodoxime } \\
\text { /clavulanic acid }\end{array}$ & Amikacine & Ciprofloxacine & Cotrimoxazol & Imipenem \\
\hline Spring & $\begin{array}{l}2 \\
(25 \%)\end{array}$ & $\begin{array}{l}2 \\
(100 \%)\end{array}$ & $\begin{array}{l}1 \\
(50 \%)\end{array}$ & $\begin{array}{l}2 \\
(100 \%)\end{array}$ & $\begin{array}{l}1 \\
(50 \%)\end{array}$ & $\begin{array}{l}0 \\
(50 \%)\end{array}$ & 1 & 0 \\
\hline Summer & $\begin{array}{l}1 \\
(12.5 \%)\end{array}$ & $\begin{array}{l}1 \\
(100 \%)\end{array}$ & 0 & $\begin{array}{l}1 \\
(100 \%)\end{array}$ & 0 & 0 & 0 & 0 \\
\hline Fall & $\begin{array}{l}2 \\
(25 \%)\end{array}$ & $\begin{array}{l}2 \\
(100 \%)\end{array}$ & $\begin{array}{l}1 \\
(50 \%)\end{array}$ & $\begin{array}{l}2 \\
(100 \%)\end{array}$ & $\begin{array}{l}1 \\
(50 \%)\end{array}$ & 0 & $\begin{array}{l}1 \\
(50 \%)\end{array}$ & 0 \\
\hline Winter & $\begin{array}{l}3 \\
(37.5 \%)\end{array}$ & $\begin{array}{l}3 \\
(100 \%)\end{array}$ & $\begin{array}{l}2 \\
(66.7 \%)\end{array}$ & 3 & $\begin{array}{l}1 \\
(33.3 \%)\end{array}$ & 0 & $\begin{array}{l}1 \\
(33.3 \%)\end{array}$ & 0 \\
\hline Total & $\begin{array}{l}8 \\
(100 \%)\end{array}$ & $\begin{array}{l}8 \\
(100 \%)\end{array}$ & $\begin{array}{l}4 \\
(50 \%)\end{array}$ & $\begin{array}{l}8 \\
(100 \%)\end{array}$ & $\begin{array}{l}3 \\
(37.5 \%)\end{array}$ & 0 & $\begin{array}{l}3 \\
(37.5 \%)\end{array}$ & 0 \\
\hline
\end{tabular}

Table 14: Confirming stage and effect of non-beta-lactam antibiotics toward K.pnemoniae producing ESBLs isolated from patients with RTI

\begin{tabular}{|c|c|c|c|c|c|c|c|c|}
\hline RTI & $\begin{array}{l}\text { K.pneumoniae } \\
\text { suspected to } \\
\text { produce ESBLs }\end{array}$ & $\begin{array}{l}\text { Ceftazidime/ } \\
\text { clavulanic acid }\end{array}$ & $\begin{array}{l}\text { Cefotaxime/ } \\
\text { clavulanic acid }\end{array}$ & $\begin{array}{l}\text { Cefpodoxime/ } \\
\text { clavulanic acid }\end{array}$ & Amikacine & Ciprofloxacine & Cotrimoxazol & Imipenem \\
\hline Spring & $\begin{array}{l}7 \\
(18.9 \%)\end{array}$ & $\begin{array}{l}6 \\
(85.7 \%)\end{array}$ & $\begin{array}{l}3 \\
(42.85 \%)\end{array}$ & $\begin{array}{l}7 \\
(100 \%)\end{array}$ & $\begin{array}{l}3 \\
(42.85 \%)\end{array}$ & $\begin{array}{l}2 \\
(28.6 \%)\end{array}$ & $\begin{array}{l}3 \\
(42.85 \%)\end{array}$ & 0 \\
\hline Summer & $\begin{array}{l}2 \\
(5.4 \%)\end{array}$ & $\begin{array}{l}2 \\
(100 \%)\end{array}$ & $\begin{array}{l}1 \\
(50 \%)\end{array}$ & $\begin{array}{l}2 \\
(100 \%)\end{array}$ & $\begin{array}{l}1 \\
(50 \%)\end{array}$ & $\begin{array}{l}1 \\
(50 \%)\end{array}$ & $\begin{array}{l}1 \\
(50 \%)\end{array}$ & 0 \\
\hline Fall & $\begin{array}{l}8 \\
(21.6 \%)\end{array}$ & $\begin{array}{l}8 \\
(100 \%)\end{array}$ & $\begin{array}{l}2 \\
(25 \%)\end{array}$ & $\begin{array}{l}8 \\
(100 \%)\end{array}$ & $\begin{array}{l}2 \\
(25 \%)\end{array}$ & $\begin{array}{l}1 \\
(12.5 \%)\end{array}$ & $\begin{array}{l}2 \\
(25 \%)\end{array}$ & 0 \\
\hline Winter & $\begin{array}{l}20 \\
(54.1 \%)\end{array}$ & $\begin{array}{l}16 \\
(80 \%)\end{array}$ & $\begin{array}{l}8 \\
(40 \%)\end{array}$ & $\begin{array}{l}20 \\
(100 \%)\end{array}$ & $\begin{array}{l}7 \\
(35 \%)\end{array}$ & $\begin{array}{l}4 \\
(20 \%)\end{array}$ & $\begin{array}{l}8 \\
(40 \%)\end{array}$ & 0 \\
\hline Total & $\begin{array}{l}37 \\
(100 \%)\end{array}$ & $\begin{array}{l}32 \\
(86.5 \%)\end{array}$ & $\begin{array}{l}14 \\
(37.9 \%)\end{array}$ & $\begin{array}{l}37 \\
(100 \%)\end{array}$ & $\begin{array}{l}13 \\
(35.1 \%)\end{array}$ & $\begin{array}{l}8 \\
(21.6 \%)\end{array}$ & $\begin{array}{l}14 \\
(37.9 \%)\end{array}$ & 0 \\
\hline
\end{tabular}

Of the thirty four K. pneumoniae with blaCTX-M , $41.2 \%(\mathrm{n}=14), 11.8 \%(\mathrm{n}=4), 11.8 \%(\mathrm{n}=4), 5.9 \%(\mathrm{n}=$ 2) and $29.5 \%(\mathrm{n}=10)$ were obtained from patients with UTI, in ICUs ward, in surgery ward, patients with lesions and respiratory tract infections, respectively. In patients with UTI, fifty seven $K$. pneumoniae with blaSHV were observed. Of these $28.1 \%(n=16), 8.7 \%(n=5), 28.1 \%$ $(\mathrm{n}=16)$ and $35.1 \%(\mathrm{n}=20)$ were obtained in spring, summer, fall and winter, respectively. Of the four $K$. pneumoniae with blaTEM, 25\% $(\mathrm{n}=1), 25 \%(\mathrm{n}=1)$ and $50 \%(\mathrm{n}=2)$ were found in spring, fall and winter, respectively. Our findings also showed that of the fourteen $K$. pneumoniae with blaCTX-M from the patients with UTIs, $7.1 \%(\mathrm{n}=1)$ were obtained in spring, $21.5 \%(\mathrm{n}=3)$ in fall and $71.4 \%(\mathrm{n}=10)$ in winter. Of the eleven $K$. pneumoniae with blaSHV from patients admitted in ICUs, $18.2 \%(\mathrm{n}=2), 9.1 \%(\mathrm{n}=1), 27.2 \%$ $(\mathrm{n}=3)$ and $45.5 \%(\mathrm{n}=5)$ were found in spring, summer, fall and winter, respectively. Two $K$. pneumoniae with blaTEM were isolated in fall and winter. Four $K$. pneumoniae with blaCTX-M were observed, of which $25 \%(\mathrm{n}=1), 25 \%(\mathrm{n}=1)$ and $50 \%$ $(\mathrm{n}=2)$ were obtained in summer, fall and winter, respectively. Of the eleven $K$. pneumoniae with blaSHV from patients admitted in the surgery ward, 9.1\% $(\mathrm{n}=1), 36.4 \%(\mathrm{n}=4), 18.2 \%(\mathrm{n}=2)$ and $36.4 \%$ $(\mathrm{n}=4)$ were obtained in spring, summer, fall and winter, respectively. Two K. pneumoniae with blaTEM were isolated, of which one was obtained in fall and the other in winter. Of the four K. pneumoniae with blaCTX-M from the surgery ward, $25 \%(\mathrm{n}=1)$ were obtained in summer, $25 \%(\mathrm{n}=1)$ in fall and $50 \%(\mathrm{n}=$ 2 ) in winter. The results showed that of the eight $K$. pneumoniae with blaSHV, from patients with lesion infections, $25 \%(\mathrm{n}=2), 12.5 \%(\mathrm{n}=1), 25 \%(\mathrm{n}=2)$ and $37.5 \%(\mathrm{n}=3)$ were found in spring, summer, fall and winter, respectively. One K.pneumoniae with blaTEM was isolated in winter. Of the two $K$. pneumoniae with blaCTX-M, from the patients with lesion infections, $50 \%(\mathrm{n}=1)$ were obtained in spring and $50 \%(\mathrm{n}=1)$ in winter.

The results indicated that of the thirty-three $K$. pneumoniae with blaSHV, from patients with RTIs, $18.8 \%(\mathrm{n}=6), 9 \%(\mathrm{n}=3), 24.2 \%(\mathrm{n}=8)$ and $48.5 \%$ $(\mathrm{n}=16)$ were obtained in spring, summer, fall and winter, respectively. Of the eight $K$. pneumoniae with blaTEM from RTIs, 37.5\% $(\mathrm{n}=3), 12.5 \%(\mathrm{n}=1), 12.5 \%(\mathrm{n}=1)$ and $37.5 \%(\mathrm{n}=3)$ were obtained in spring, summer, fall and winter, respectively. Of the ten K. pneumoniae with blaCTX-M, from patients with RTIs, $30 \%(\mathrm{n}=3)$ were obtained in spring, $10 \%(\mathrm{n}=1)$ in summer, $10 \%(\mathrm{n}=1)$ in fall and $50 \%(\mathrm{n}=5)$, in winter. 


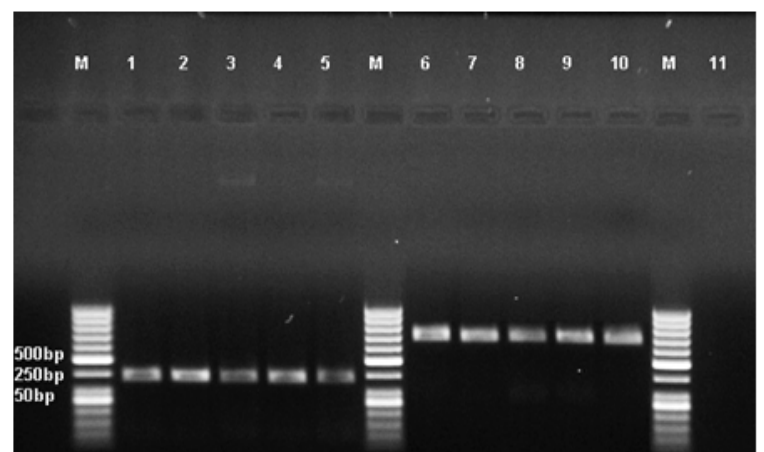

Fig. 1: Agarose gel electropherosis of amplified fragment obtained by PCR for blaSHV and blaCTX-M. Lanes: $\mathrm{M}=$ Molecular weight marker (50bp) $1=$ K.pneumoniae 7881 (positive control), 2-5 blaSHV, 6-10 BlaCTX$\mathrm{M}, 11=$ negative control

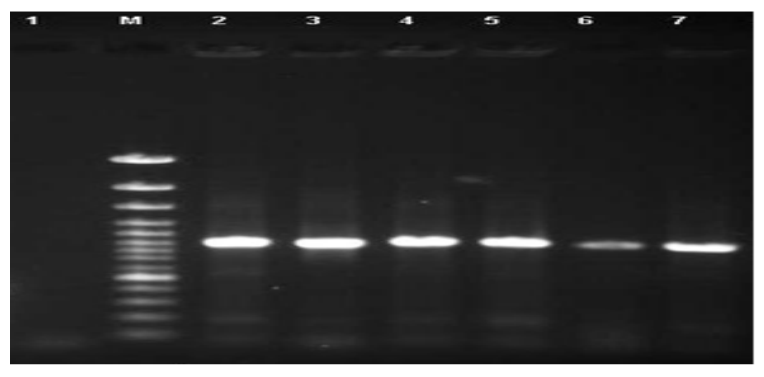

Fig. 2: Agarose gel electropherosis of amplified fragment obtained by PCR for blaTEM. Lanes: 1 = Negative control $\mathrm{M}=$ Molecular weight marker (100bp) $2=$ K.pneumoniae 7881 (positive control), 3-7 blaTEM.

The results reflected the frequency of blaSHV, blaTEM and blaCTX-M among K.pneumoniae producing ESBLs were 87.5, 12.4 and 24.8\%, respectively. Further, 1.5, 7.9, 0.4 and $1.5 \%$ of K.pneumoniae producing ESBLs were found positive for blaSHV-TEM, blaSHV-CTX-M, blaTEM-CTX-M and blaSHV-TEM-CTX-M, respectively.

blaSHV, TEM and CTX-M showed in Fig. 1-2.

MLST results: MLST was assessed for thirty $K$. pneumoniae producing ESBLS in Milad hospital, of which thirteen had blaTEM, BlaSHV, seven blaSHV and blaCTX-M, two blaSHV and the other eight blaTEM, BlaSHV and blaCTX-M. The MLST data calculated by eBURST were the Sequence Types (STs) and their associated allelic profiles. The different allelic profiles are showed in Table 16. The Sequence Type (ST) was designated for each unique allelic profile. The definition of the group is to identify groups of related STs using the most conservative definition, where all members assigned to the same group share identical alleles at $=4$ of the 5 loci, with at least one other member of the group.

Clonal complex is a set of STs that are all believed to have descended from the same founding genotype. Using the stringent group definition (4/5 shared alleles) isolates in the group defined by eBURST will be considered to belong to a single clonal complex.

$\mathrm{SLV}=$ all STs must be a Single Locus Variant (SLV) of at least one other ST in the group

$\mathrm{DLV}=$ double locus variant of at least one other ST in the group.

$\mathrm{TLV}=$ three loci (TLVs)and those that are more distantly related (satellites).

The bootstrap values shown for each ST are the percentage of times the ST was predicted to be the primary founder of the group in the bootstrap resamplings. As a ST cannot be the predicted founder if it is not present in a re-sampled dataset, the calculation of the percentage of times each ST is predicted to be the primary founder omits those re-samplings in which that ST is absent. In larger eBURST groups there may be several STs besides the predicted primary founder that have a number of SLVs of their own. A ST that appears to have diversified to produce multiple SLVs was called a subgroup founder (eBURST program).

Based on the nucleotide variations of the five genetic loci, twenty-five different STs could be identified among thirty K.pneumoniae producing ESBLs isolates. The majority of these (5 out of $25 \mathrm{STs}$ ) were represented by a single isolate. Among the STs shared by multiple isolates, the most frequently encountered were ST14 (four isolates) ST16 (two isolates), ST18 (two isolates). Six Colonal Complexes (CCs) were identified. The predicted founder was defined in the eBURST results. $\mathrm{CC} 1$ included five STs (st1,5,4,3,2), CC2 three STs (ST9,10,11), CC3 three STs (ST8,7,6), CC4 two STs (st12,13),CC5 two STs $(\mathrm{ST} 15,14)$ and CC6 two STs $(\mathrm{ST} 16,17)$ (Table 15).

K.oxytoca: The results showed that fifteen clinical isolates of K.oxytoca were obtained in the surgery ward, patients with lesion and respiratory tract infections. It was found that $40 \%(n=6)$ and $27.3 \%(n=3)$ of K.oxytoca producing ESBLs were resistant to cotrimoxazol and amikacin, respectively. Resistance to the other non-beta-lactam antibiotics were not observed (Table 17). The findings showed $73.3 \%$ of K.oxytoca produces ESBLs. 
Am. J. Infect. Dis., 6 (4): 110-121, 2010

eBURST results was:

eBURST Report

No. isolates $=25 \mid$ No. STs $=25 \mid$ No. re-samplings for bootstrapping $=1000$

No. loci per isolate $=5 \mid$ No. identical loci for group def $=4 \mid$ No. groups $=6$

Group 1: No. Isolates $=5 \mid$ No. STs $=5 \mid$ Predicted Founder $=3$

\begin{tabular}{|c|c|c|c|c|c|c|c|c|}
\hline ST & FREQ & SLV & $\begin{array}{l}\text { Average } \\
\text { DLV }\end{array}$ & $\begin{array}{l}\text { ST Bootstrap } \\
\text { TLV }\end{array}$ & SAT & Distance & Group & Subg \\
\hline k4 & 1 & 3 & 1 & 0 & 0 & 1.25 & $60 \%$ & $16 \%$ \\
\hline $\mathrm{k} 26$ & 1 & 2 & 2 & 0 & 0 & 1.5 & $19 \%$ & $0 \%$ \\
\hline k19 & 1 & 1 & 2 & 1 & 0 & 2.0 & $0 \%$ & $0 \%$ \\
\hline $\mathrm{k} 7$ & 1 & 1 & 2 & 1 & 0 & 2.0 & $0 \%$ & $0 \%$ \\
\hline k10 & 1 & 1 & 1 & 2 & 0 & 2.25 & $0 \%$ & $0 \%$ \\
\hline
\end{tabular}

Group 2: No. Isolates $=3 \mid$ No. STs $=3 \mid$ Predicted Founder $=$ Multiple Candidates

$\begin{array}{lllll} & & & \text { Average } & \text { ST Bootstrap } \\ \text { ST } & \text { FREQ } & \text { SLV } & \text { DLV } & \text { TLV } \\ \text { k20 } & 1 & 2 & 0 & 0 \\ \text { k1 } & 1 & 2 & 0 & 0 \\ \text { k23 } & 1 & 2 & 0 & 0\end{array}$

Group 3: No. Isolates $=3 \mid$ No. STs $=3 \mid$ Predicted Founder $=13$

$\begin{array}{llll} & & & \text { Average } \\ \text { ST } & \text { FREQ } & \text { SLV } & \text { DLV } \\ \text { k15 } & 1 & 2 & 0 \\ \text { k16 } & 1 & 1 & 1 \\ \text { k14 } & 1 & 1 & 1\end{array}$

Group 4: No. Isolates $=2 \mid$ No. STs $=2 \mid$ Predicted Founder $=$ None

\begin{tabular}{|c|c|c|c|c|c|c|}
\hline ST & FREQ & SLV & DLV & TLV & SAT & Distance \\
\hline $\mathrm{k} 13$ & 1 & 1 & 0 & 0 & 0 & 1.0 \\
\hline k12 & 1 & 1 & 0 & 0 & 0 & 1.0 \\
\hline \multicolumn{7}{|c|}{ Group 5: No. Isolates $=2 \mid$ No. STs $=2 \mid$ Predicted Founder $=$ None } \\
\hline ST & FREQ & SLV & DLV & TLV & SAT & Distance \\
\hline k8 & 1 & 1 & 0 & 0 & 0 & 1.0 \\
\hline & 1 & 0 & 0 & 0 & 1.0 & \\
\hline \multicolumn{7}{|c|}{ Group 6: No. Isolates $=2 \mid$ No. STs $=2 \mid$ Predicted Founder $=$ None } \\
\hline ST & FREQ & SLV & DLV & TLV & SAT & Distance \\
\hline k6 & 1 & 1 & 0 & 0 & 0 & 1.0 \\
\hline k5 & 1 & 1 & 0 & 0 & 0 & 1.0 \\
\hline
\end{tabular}

Singletons: size 8

$\mathrm{k} 21, \mathrm{k} 18, \mathrm{k} 17, \mathrm{k} 11, \mathrm{k} 28, \mathrm{k} 2, \mathrm{k} 25, \mathrm{k} 24$

Screening stage: $K$. oxytoca was only found in winter from patients admitted in the surgery ward (Table 18). The findings indicated that one K. oxytoca obtained from patients with lesion infections in autumn, was resistant to all the third-generation of cephalosprines, except cefotaxime (Table 19). Of the twelve $K$. oxytoca collected from patients with RTIs, $16.6 \%(\mathrm{n}=$ 2) were obtained in fall and $83.3 \%(\mathrm{n}=10)$ in winter (Table 20).

Confirming stage: Two K. oxytoca were isolated from patients admitted in surgery wards in winterand both were suspected of being able to produce ESBLs. They were confirmed by ceftazidim/clavulanic acid and cefpodoxim/clavulanic acid (Table 21). One K. oxytoca, collected from patients with lesion infections in fall, was confirmed by ceftazidime/clavulanic acid as well as cefpodoxime/clavulanic acid, at the confirming stage, to be prone to produce ESBLs (Table 22). The two $K$. oxytoca collected from patients with RTIs in fall, were confirmed by ceftazidime/clavulanic acid and cefpodoxime/clavulanic acid, to produce ESBLs. Of the ten K.oxytoca collected from patients with RTIs in winter, $60 \%(\mathrm{n}=6)$ were suspected of being able to produce ESBLs. They were confirmed by ceftazidime/clavulanic acid and cefpodoxime/clavulanic acid, at the confirming stage (Table 23).

PCR results: Of the eleven $K$. oxytoca producing ESBLs at the phenotypic stage, $100 \%(\mathrm{n}=11)$ blaSHV were obtained by the PCR method. The results that showed blaTEM and blaCTX-M were negative. 
Am. J. Infect. Dis., 6 (4): 110-121, 2010

Table 15: Characteristics of 30 ESBL-producing K. pneumoniae isolates from different parts of Milad hospital

\begin{tabular}{|c|c|c|c|c|c|c|c|}
\hline & Allelic profile & $\mathrm{St}$ & Part of hospital & $\mathrm{cc}$ & blaSHV & blaTEM & BlaCTX-M \\
\hline \multirow[t]{2}{*}{1} & $1-6-3-5-6-9$ & & & & & & \\
\hline & 7 & ICUs & 3 & 5 & 12 & - & \\
\hline 2 & $1-3-1-5-4$ & 23 & UTI & - & 12 & 12 & 15 \\
\hline \multirow[t]{2}{*}{3} & $4-4-2-4-3$ & & & & & & \\
\hline & 16 & LESION & 6 & 12 & 12 & - & \\
\hline 4 & $2-2-6-3-10$ & 1 & UTI & 1 & 12 & 12 & 15 \\
\hline 5 & $4-2-2-4-3$ & 17 & RTI & 5 & 12 & 12 & - \\
\hline \multirow[t]{2}{*}{6} & $4-4-2-4-3$ & & & & & & \\
\hline & 16 & SURGERY & 6 & 12 & 12 & 15 & \\
\hline \multirow[t]{2}{*}{7} & $1-2-6-3-10$ & & & & & & \\
\hline & 4 & UTI & 1 & 12 & 12 & 15 & \\
\hline 8 & $2-1-2-4-2$ & 14 & RTI & 5 & 12 & 12 & 15 \\
\hline 9 & $2-1-2-4-2$ & 14 & RTI & 5 & 12 & 12 & 15 \\
\hline \multirow[t]{2}{*}{10} & $2-2-5-3-9$ & & & & & & \\
\hline & 5 & UTI & 1 & 12 & 12 & - & \\
\hline 11 & $5-4-2-4-1$ & 21 & RTI & - & 5 & 12 & - \\
\hline 12 & $2-7-4-1-10$ & 13 & SURGERY & 4 & 12 & 12 & 15 \\
\hline 13 & $2-9-4-1-10$ & 12 & RTI & 4 & 12 & 12 & 15 \\
\hline 14 & $2-2-4-4-7$ & 11 & RTI & 2 & 12 & - & 15 \\
\hline \multirow[t]{2}{*}{15} & $2-2-4-4-8$ & & & & & & \\
\hline & 9 & SURGERY & 2 & 12 & - & 15 & \\
\hline 16 & $2-2-3-4-8$ & 10 & RTI & 2 & 12 & - & - \\
\hline 17 & $2-8-3-4-5$ & 20 & ICUs & - & 12 & 12 & - \\
\hline \multirow[t]{2}{*}{18} & $1-10-2-4-6$ & & & & & & \\
\hline & 19 & LESION & - & 5 & 12 & - & \\
\hline 19 & $2-4-6-3-10$ & 3 & UTI & 1 & 12 & - & - \\
\hline \multirow[t]{2}{*}{20} & $6-11-5-6-9$ & & & & & & \\
\hline & 6 & RTI & 3 & 12 & 12 & - & \\
\hline 21 & $4-1-1-1-4$ & 18 & SURGERY & - & 12 & - & - \\
\hline 22 & $4-1-1-1-4$ & 18 & SURGERY & - & 12 & 12 & - \\
\hline 23 & $6-2-5-6-9$ & 8 & ICUs & 3 & 12 & 12 & - \\
\hline \multirow[t]{2}{*}{24} & $1-1-3-4-2$ & & & & & & \\
\hline & 25 & RTI & - & 12 & - & - & \\
\hline \multirow[t]{2}{*}{25} & $2-6-2-2-3$ & & & & & & \\
\hline & 24 & RTI & - & 12 & 12 & - & \\
\hline 26 & $2-2-5-3-10$ & 2 & UTI & 1 & 12 & 12 & - \\
\hline \multirow[t]{2}{*}{27} & $2-1-5-4-2$ & & & & & & \\
\hline & 15 & RTI & 5 & 12 & 12 & - & \\
\hline \multirow[t]{2}{*}{28} & $2-3-3-4-2$ & & & & & & \\
\hline & 22 & UTI & - & 5 & & - & \\
\hline 29 & $2-1-2-4-2$ & 14 & RTI & 5 & 12 & & - \\
\hline 30 & $2-1-2-4-2$ & 14 & RTI & 5 & 12 & & - \\
\hline
\end{tabular}

Table 16: Variation in loci used in the present K. pneumoniae MLST scheme

\begin{tabular}{lll}
\hline Locus & Size & No. Allele \\
\hline Gapa & $366 \mathrm{bp}$ & 6 \\
Gyra & $752 \mathrm{bp}$ & 11 \\
Gyrb & $648 \mathrm{bp}$ & 5 \\
Rpob & $687 \mathrm{bp}$ & 6 \\
Groel & $786 \mathrm{bp}$ & 10 \\
\hline
\end{tabular}

Table 17: Effect of non-beta-lactam antibiotics against K.oxytoca producing ESBLs

\begin{tabular}{lllll}
\hline & K.oxytoca producing ESBLs & Amikacine & Ciprofloxacine & Cotrimoxazol \\
\hline Total & 11 & 3 & 0 & 6 \\
& $(100 \%)$ & $(27.3 \%)$ & & $(54.5 \%)$ \\
\hline
\end{tabular}

Table 18: Screening stage for detection of K.oxytoca producing ESBLs from patients in surgery ward

\begin{tabular}{lllllll}
\hline Surgery ward & K.oxytoca & Ceftazidime & Cefotaxime & Cefteryaxone & Cefpodoxime & Aztreonam \\
\hline Winter & 2 & 2 & 2 & 2 & 2 & 2 \\
& $(100 \%)$ & $(100 \%)$ & $(100 \%)$ & $(100 \%)$ & $(100 \%)$ & $(100 \%)$ \\
\hline
\end{tabular}


Am. J. Infect. Dis., 6 (4): 110-121, 2010

Table 19: Screening stage for detection of K.oxytoca producing ESBLs from patients with lesion infection

\begin{tabular}{lllllll}
\hline Lesion infection & K.oxytoca & Ceftazidime & Cefotaxime & Cefteryaxone & Cefpodoxime & Aztreoname \\
\hline Fall & 1 & 1 & 0 & 1 & 1 & 1 \\
& $(100 \%)$ & $(100 \%)$ & & $(100 \%)$ & $(100 \%)$ & $(100 \%)$ \\
\hline
\end{tabular}

Table 20: Screening stage for detection of K.oxytoca producing ESBLs from patients with RTI

\begin{tabular}{|c|c|c|c|c|c|c|}
\hline RTI & K.Oxytoca & Ceftazidime & Cefotaxime & Cefteryaxone & Cefpodoxime & Aztreonam \\
\hline Fall & $\begin{array}{l}2 \\
(16.6 \%)\end{array}$ & $\begin{array}{l}2 \\
(100 \%)\end{array}$ & 0 & $\begin{array}{l}2 \\
(100 \%)\end{array}$ & $\begin{array}{l}2 \\
(100 \%)\end{array}$ & $\begin{array}{l}2 \\
(100 \%)\end{array}$ \\
\hline Winter & $\begin{array}{l}10 \\
(83.3 \%)\end{array}$ & $\begin{array}{l}8 \\
(80 \%)\end{array}$ & $\begin{array}{l}4 \\
(40 \%)\end{array}$ & $\begin{array}{l}7 \\
(70 \%)\end{array}$ & $\begin{array}{l}6 \\
(60 \%)\end{array}$ & $\begin{array}{l}6 \\
(60 \%)\end{array}$ \\
\hline Total & $\begin{array}{l}12 \\
(100 \%)\end{array}$ & $\begin{array}{l}10 \\
(83.3 \%)\end{array}$ & $\begin{array}{l}4 \\
(33.3 \%)\end{array}$ & $\begin{array}{l}9 \\
(75 \%)\end{array}$ & $\begin{array}{l}8 \\
(66.7 \%)\end{array}$ & $\begin{array}{l}8 \\
(66.7 \%)\end{array}$ \\
\hline
\end{tabular}

Table 21: Confirming stage and effect of non-beta-lactam antibiotics toward K.oxytoca producing ESBLs isolated from patients in surgery ward

\begin{tabular}{llllllll}
\hline Surgery ward & $\begin{array}{l}\text { K.oxytoca } \\
\text { suspectedto } \\
\text { produce ESBLs }\end{array}$ & $\begin{array}{l}\text { Ceftazidime } \\
\text { /clavulanic acid/clavulanic acid/clavulanic acid }\end{array}$ & $\begin{array}{l}\text { Cefotaxime } \\
\text { Cefpodoxime }\end{array}$ & Amikacine & Ciprofloxacine & Cotrimoxazol & Imipenem \\
\hline Winter & 2 & 2 & 0 & 2 & 0 & 0 & 1 \\
& $(100 \%)$ & $(100 \%)$ & 0 & $(100 \%)$ & 0 & 0 & $(50 \%)$ \\
\hline
\end{tabular}

Table 22: Confirming stage and effect of non-beta-lactam antibiotics toward K.oxytoca producing ESBLs isolated from patients with Lesion infection

\begin{tabular}{|c|c|c|c|c|c|c|c|c|}
\hline Lesion infection & $\begin{array}{l}\text { K.oxytoca } \\
\text { suspected to } \\
\text { produce ESBLS }\end{array}$ & $\begin{array}{l}\text { Ceftazidime } \\
\text { /clavulanic acid }\end{array}$ & $\begin{array}{l}\text { Cefotaxime } \\
\text { /clavulanic acid }\end{array}$ & $\begin{array}{l}\text { Cefpodoxime } \\
\text { /clavulanic acid }\end{array}$ & Amikacine & Ciprofloxacine & Cotrimoxazol & Imipenem \\
\hline Fall & $\begin{array}{l}1 \\
(100 \%)\end{array}$ & $\begin{array}{l}1 \\
(100 \%)\end{array}$ & $\begin{array}{l}0 \\
0\end{array}$ & $\begin{array}{l}1 \\
(100 \%)\end{array}$ & $\begin{array}{l}0 \\
0\end{array}$ & $\begin{array}{l}0 \\
0\end{array}$ & $\begin{array}{l}0 \\
0\end{array}$ & $\begin{array}{l}0 \\
0\end{array}$ \\
\hline
\end{tabular}

Table 23: Confirming stage and effect of non-beta-lactam antibiotics toward K.oxytoca producing ESBLs isolated from patients with RTI

\begin{tabular}{|c|c|c|c|c|c|c|c|c|}
\hline RTI & $\begin{array}{l}\text { K.oxytoca } \\
\text { suspected to } \\
\text { produce ESBLs }\end{array}$ & $\begin{array}{l}\text { Ceftazidime } \\
\text { /clavulanic acid }\end{array}$ & $\begin{array}{l}\text { Cefotaxime } \\
\text { /clavulanic acid }\end{array}$ & $\begin{array}{l}\text { Cefpodoxime } \\
\text { /clavulanic acid }\end{array}$ & Amikacine & Ciprofloxacine & Cotrimoxazol & Imipenem \\
\hline Fall & $\begin{array}{l}2 \\
(25 \%)\end{array}$ & $\begin{array}{l}2 \\
(100 \%)\end{array}$ & 0 & $\begin{array}{l}2 \\
(100 \%)\end{array}$ & $\begin{array}{l}1 \\
(50 \%)\end{array}$ & 0 & $\begin{array}{l}1 \\
(50 \%)\end{array}$ & 0 \\
\hline Winter & $\begin{array}{l}6 \\
(75 \%)\end{array}$ & 6 & 0 & $\begin{array}{l}6 \\
(100 \%)\end{array}$ & 2 & 0 & $\begin{array}{l}4 \\
(66.6 \%)\end{array}$ & 0 \\
\hline Total & $\begin{array}{l}8 \\
(100 \%)\end{array}$ & $\begin{array}{l}8 \\
(100 \%)\end{array}$ & 0 & $\begin{array}{l}8 \\
(100 \%)\end{array}$ & $\begin{array}{l}3 \\
(37.5 \%)\end{array}$ & 0 & $\begin{array}{l}5 \\
(62.5 \%)\end{array}$ & 0 \\
\hline
\end{tabular}

\section{DISCUSSION}

Gram-negative pathogens harboring ESBLs have caused numerous outbreaks of infections and are becoming an increasing therapeutic problem in many countries. The incidence of ESBL-producing strains among clinical isolates has been steadily increasing over the past years resulting in limitations of therapeutic option (AL-Haj et al., 2010). ESBLs are now a significant problem in hospitalized patients throughout the world. The prevalence of ESBLs among clinical isolates varies worldwide and patterns are rapidly changing over time (Shayanfar et al., 2010).

These findings showed high level of antibiotic resistance, especially, for third-generation of cephalosporins. Interestingly, we found Imipenem to be effective in this study and could still be used for treatment. However our findings revealed the urgency for controlling irregular consumption of antibiotics in Iran.
K.pneumoniae: The highest resistance was found for ceftazidime $(65.8 \%)$ at the screening stage (Table 3) and The ESBLs production by K.pneumoniae from patients with UTI $(49.6 \%)$ was more than in the others. Cotrimoxazol resistance was the highest due to nonbeta-lactam antibiotics (Table 4). Ceftazidime and cefteriaxone resistance were the highest in patients with UTI at the screening stage (Table 5). In these patients, the highest K.pneumoniae producing ESBLs (42.65\%) and amikacin resistance was found in winter $(41.4 \%)$ while resistance to ciprofloxacin $(27.8 \%)$ and cotrimoxazol $(50 \%)$ was more in fall than in the other seasons (Table 10). The results showed that blaSHV was the dominant gene responsible for ESBLs production, followed by blaSHV and blaCTX-M. Frequency of all the three genes in winter was more than in the other seasons while the lowest frequency wasin summer. Our findings showed resistance to 
antibiotics and K.pneumoniae producing ESBLs in the cold seasons was higher than during the warm seasons.

In ICUs, the highest resistance to third-generation of cephalosporins was observed for cefotaxime (83.4\%) in spring (Table 6). K.pneumoniae producing ESBLs was more in winter than in the other seasons $(38.4 \%)$. $\%)$. Also the highest ciprofloxacin resistance was reported in winter $(60 \%)$ while amikacin and cotrimoxazol resistance was more in spring than in the other seasons $(50 \%)$ (Table 11$)$. The results indicated that the dominant gene was blaSHV and the highest blaSHV was observed in winter, followed by blaSHV and blaCTX-M, which showed greater frequency than blaTEM (Table 7). Our results from patients admitted in surgery wards indicated that ceftazidime resistance in summer and winter $(100 \%)$ was the highest compared to other antibiotics at the screening stage. The findings of this study also clarified that there were more K.pneumoniae producing ESBLs in winter than in the other seasons $(42.65 \%)$. We found cotrimoxazol $(50 \%)$ and ciprofloxacin $(27.8 \%)$ resistance in fall to be more than in the other seasons while the highest amikacin resistance occurred in winter (41.4\%) (Table 12). BlaSHV was the dominant gene responsible for ESBLs production but it showed the same frequency in winter and summer while blaTEM in fall and winter.

K.pneumoniae isolated from patients with lesion infections were more resistant to ceftazidime in winter $(87.5 \%)$ at the screening stage (Table 8$)$. We also found winter as a season responsible for the highest ESBLs production (37.5\%). Cotrimoxazol and amikacin resistance in spring and fall $(50 \%)$ were more than in the other seasons (Table 13). The results further showed that the dominant gene responsible for ESBLs production was blaSHV. The results showed that for patients with RTIs, the highest antibiotic resistance at the screening stage was observed for ceftazidime (91.7\%) in fall (Table 9). However ESBLs production in winter $(54.1 \%)$ was greater than in the other seasons. Amikacin, cotrimoxazol and ciprofloxacin resistance were observed to be more in summer (50\%) (Table 14). According to the findings of this study, the dominant gene responsible for ESBLs production was BlaSHV and the highest frequency of this gene was found in winter. The frequency of blaTEM and blaCTX-M in winter were also more than in the other seasons.

The highest resistance to cotrimoxazol was found in K.oxytoca producing ESBLs isolated from patients with RTIs in winter (Table 17). Ciprofloxacin and imipenem were found to be effective antibiotics in this study. Our study showed that antimicrobial resistance in $K$. pneumoniae was higher than in $K$. oxytoca but frequency of ESBLs due to $K$. oxytoca $(73.3 \%)$ was more than K. pneumoniae (51.6\%). Frequency of the blaSHV was more in comparison with the other genes responsible for ESBLs production. The results showed that $K$. oxytoca only had frequency in cold seasons. Our findings showed the dominant gene responsible for ESBLs production was blaSHV and it had the highest frequency in winter. Generally, resistance to thirdgeneration cephalosporins and aztreonam and ESBLs production in cold seasons was greater than during the warm seasons. These results were valid for resistance of $K$. pneumoniae producing ESBLs to non-beta-lactam antibiotics. It also showed that all isolates from all parts were susceptible to imipenem.

Molecular typing is a pre-requisite for elucidating the epidemiology and population structure of bacterial pathogens. Of the molecular typing methods, MLST is becoming more popular, owing to its several advantages, which have been discussed repeatedly elsewhere (like PFGE, RFFLP). It enjoys a high level of discrimination, unambiguity, reproducibility and scalability due to application of nucleotide sequences and also has an electronic portability via the Internet, so that it can easily analyze the generated data with a wider applicability (Andrade et al., 2010).

The objectives of this study were to detect the dominant colonal type by MLST. The dominant Sequence Type (ST) was ST14. The findings of this study showed different colonal complex (CC). Interestingly, most STs (ST14) were observed in RTIs. In UTI, there were no CC and the isolates were from different STs. Our findings showed a different ST and reduced epidemiology of the ESBLs in Milad Hospital. This was the first study of MLST scheme of K.pneumoniae producing ESBLs in Iran. The results of this study indicated that the most genes were BlaSHV12. The highest allelic variation occurred in GyrA (11allele).

One hundred and sixty eight clinical isolates of K.pneumoniae were collected in a survey during the period September 2006 to February 2007, from three general hospitals in Tehran, Iran. It was found that $69 \%$ of the one hundred and sixty eight clinical isolates were positive and fifty-one isolates $(31 \%)$ were negative for ESBLs (Bameri et al., 2010). Our results showed lower frequency of ESBL production in K.pneumoniae and higher for K.oxytoca in comparison with the study by Bameri in Milad hospital. However it was at least double when compared with Irajian et al in Semnan with 28.9\% ESBL production. (Irajian and Moghadas, 2010). In a research in Netherlands ESBLs were found in $2.4 \%$ of K.oxytoca (Strum et al., 2010) that was not 
comparable with the $73.4 \%$ ESBls positive K.oxytoca in this study. All the findings in our research showed high levels of ESBLs production by K.oxytoca.

\section{CONCLUSION}

In conclusion, the percentage of K.oxytoca producing ESBLs was higher than K.pneumoniae producing ESBLs. Generally, K.penomoniae produces more ESBLs in winter and fall than in the other seasons. The dominant gene responsible for ESBLs production was blaSHV. MLST due to K.pneumoniae producing ESBLs released different ST and different $\mathrm{CC}$, which showed that the source of ESBLs production among K.pneumoniae was not the same.

In general, resistance to any antibiotics used in this study during winter and fall were higher than in the other seasons.

\section{ACKNOWLEDGMENT}

All researchers in this manuscript are agree with the content of the manuscript and were contributed in this study. University Putra Malaysia and Ilam University of medical sciences provided partial support for the laboratory studies and interpretation.

\section{REFERENCES}

AL-Haj, N.A., N.I. Mashan, M.N. Shamsudin, H. Mohamad and C.S. Vairappan et al., 2010. Antibacterial activity of marine source extracts against multidrug resistance organisms. Am. J. Pharmacol. Toxicol., 5: 95-102. DOI: 10.3844/ajptsp.2010.95.102

Andrade, L.N., L.A.R. Minarini, A. Pitondo-Silva, E.C. Clímaco and I.C.V. Palazzo et al., 2010. Determinants of beta-lactam resistance in meningitis-causing enterobacteriaceae in Brazil. Can. J. Microbiol. 56: 399-407.

Bameri, Z., M. Chitsaz and P. Owlia, 2010. Detection of CTX-M- $\beta$ lactamases in Isolated Klebsiella pneumoniae. Iranian J. Pathol., 5: 137-142.

Erum, K., M. Ejaz, A. Zafar, K.Jabeen and S. Shakoor et al., 2010. Increased isolation of ESBL producing Klebsiella pneumoniae with emergence of carbapenem resistant isolates in Pakistan: Report from a tertiary care hospital. J Pak Med Assoc., 60: 186-190. PMID: 20225774
Irajian, G. and A.J. Moghadas, 2010. Frequency of extended-spectrum beta lactamase positive and multidrug resistance pattern in Gram-negative urinary isolates, Semnan, Iran. Jundishapur J. Microbiol., 3: 107-113.

Kamatchi, C., H. Magesh, U. Sekhar and R. Vaidyanathan, 2009. Identification of clonal clusters of Klebsiella pneumoniae Isolates from chennai by extended spectrum beta lactamase genotyping and antibiotic resistance phenotyping analysis. Am. J. Infect. Dis., 5: 74-82. DOI: 10.3844/ajidsp.2009.74.82

Mansouri, M. and R. Ramazanzadeh, 2009. Speared of extended spectrum beta-lactamases producing E.coli clinical isolates in sanandaj hospital. J. Biological Sci., 9: 362-366. DOI: 10.3923/jbs.2009.362.366

Mollet, C., M. Drancourt and D. Raoult, 1997. RpoB sequence analysis as a novel basis for bacterial identification. Mol. Microbiol., 26: 1005-11. DOI: 10.1046/j.1365-2958.1997.6382009.x

Nasehi, L, F. Shahcheraghi, V.S. Nikbin and S. Nematzadeh, 2010. PER, CTX-M, TEM and SHV Betalactamases in Clinical Isolates of Klebsiella pneumoniae Isolated from Tehran, Iran. Iranian J. Basic Med. Sci., 13: 111-118.

Shayanfar, N., M. Rezaei, M. Ahmadi, F. Ehsanipour, 2010. Evaluation of Extended Spectrum Betalactamase (ESBL) positive strains of Klebsiella pneumoniae and escherichia coli in bacterial cultures. Iranian J. Pathol., 5: 34-39.

Strum, P.D.J., E.T.M. Bochum, S.V.M. van Mook-Vermulst, C. Handgraaf and T. Klaassen et al., 2010. Prevalence, molecular characterization and phenotypic confirmation of extended-spectrum beta-lactamases in Escherichia coli, Klebsiella pneumoniae and Klebsiella oxytoca at the Radboud University Nijmegen Medical Centre in The Netherlands. Microb. Drug Resist., 16: 55-60. DOI: $10.1089 / \mathrm{mdr} .2009 .0107$

Wertz, J.E., C. Goldstone, D.M. Gordon and M.A. Riley, 2003. A molecular phylogeny of enteric bacteria and implications for a bacterial species concept. J. Evol. Biol., 16: 1236-48. DOI: 10.1046/j.14209101.2003.00612.x 